Atıf için / For Citation: V. Bozkurt, S. Ertürk, E. Şahin, "Yeni geliştirilen NUMEXO2 dijital elektronik ile EXOGAM2 dedektörünün K-Parametresine bağl1 enerji çözünürlüğ̈̈”, Süleyman Demirel Üniversitesi Fen Edebiyat Fakültesi Fen Dergisi, 15(2), 237-243, 2020.

\title{
Yeni Geliştirilen NUMEXO2 Dijital Elektronik ile EXOGAM2 Dedektörünün K- Parametresine Bağlı Enerji Çözünürlüğü
}

\author{
Vakkas BOZKURT $^{* 1}$, Sefa ERTÜRK ${ }^{1}$, Elif ŞAHINN²,a

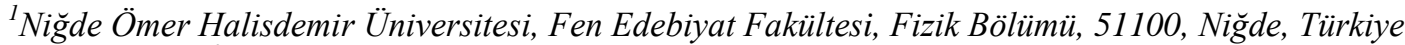 \\ ${ }^{2}$ Izmir Yüksek Teknoloji Enstitüsü, Fizik Bölümü, 35430, İzmir, Türkiye \\ ${ }^{a}$ Technische Universität Darmstadt, Institut für Kernphysik, Darmstadt, Germany \\ *yazışllan yazar e-posta: vakkasbozkurt@ohu.edu.tr
}

(Alınlş / Received: 23.09.2020, Kabul / Accepted: 03.11.2020, Yayımlanma / Published: 29.11.2020)

\begin{abstract}
Özet: $\mathrm{Bu}$ çalışmada, yeni geliştirilen dijital elektronik NUMEXO2 ile EXOGAM2(EXOtic GAMma array spectrometer) dedektörünün K-Parametrelerine (sayım oranını optimize eden Trapezoidal filtre sistemi) bağlı enerji çözünürlüğ̈̈ için ${ }^{152}$ Eu radyoaktif kaynak kullanılarak ölçüm ve analiz yapılmıştır. Yapılan bu deneysel çalışma Fransa'da bulunan GANIL (Grand Accelerature National d'Ions Lourd) nükleer araştırma merkezinde gerçekleştirilmiştir. Düşük ve yüksek gama enerji seviyesine sahip ${ }^{152} \mathrm{Eu}$ radyoaktif kaynak kullanılarak farklı K-parametreleri için gerçekleştirilen ölçüm ve analiz sonuçları iki EXOGAM2 dedektörü için gerçekleştirilmiştir. Herhangi bir K-parametresi uygulamadan birinci EXOGAM2 dedektörü için FWHM ile ifade edilen çözünürlük değerleri sırası ile $(344,278 \mathrm{keV})$ için 2,357, $(1408,006 \mathrm{keV})$ için 2,820 olarak ölçülürken, ikinci EXOGAM2 dedektörü için ise sırasıyla 2,451 ve 2,975 olarak ölçülmüştür. Ayn1 yöntem kullanılarak farklı K-Parametrelerine $(\mathrm{K}=2 \mu \mathrm{s}, \mathrm{K}=5 \mu \mathrm{s}$ ve $\mathrm{K}=10 \mu \mathrm{s})$ bağlı olarak EXOGAM2 dedektörleri için enerji çözünürlükleri elde edilmiştir.
\end{abstract}

Anahtar kelimeler: Enerji çözünürlügü, Dijital elektronik, NUMEXO2, Enerji kalibrasyonu

\section{Energy Resolution Dependent on K-parameter of EXOGAM2 Detector with Newly Developed NUMEXO2 Digital Electronics}

\begin{abstract}
In this study, energy resolution dependent on K-Parameters (Trapezoidal filter system optimizing the count rate) of EXOGAM2 detector with newly developed NUMEXO2 digital electronics using ${ }^{152} \mathrm{Eu}$ radioactive source measured and analysed. This experimental study was carried out at the GANIL (Grand Accelerature National d'Ions Lourd) nuclear research center in France. The measurement and analysis results for different $\mathrm{K}$ parameters using a ${ }^{152} \mathrm{Eu}$ radioactive source with low and high gamma energy levels were performed for two EXOGAM2 detectors. Without applying any $\mathrm{K}$ parameter, the resolution values expressed by FWHM for the first EXOGAM2 detector are measured as 2.360 for $(344.278 \mathrm{keV}), 2.820$ for $(1408.006 \mathrm{keV})$ and 2.451 and 2.975 for the second EXOGAM2 detector, respectively. By using the same procedures energy resolutions of EXOGAM2 detectors obtained for different $K$-Parameters $(K=2 \mu \mathrm{s}, K=5 \mu \mathrm{s}$ and $\mathrm{K}=10 \mu \mathrm{s})$.
\end{abstract}

Key words: Energy resolution, Digital electronics, NUMEXO2, Energy calibration

\footnotetext{
Vakkas BOZKURT, vakkasbozkurt@ohu.edu.tr, ORCID: https://orcid.org/0000-0003-4651-0447 Sefa ERTÜRK, sefa@ohu.edu.tr, ORCID: https://orcid.org/0000-0003-4051-9096 Elif ŞAHIN, ecelifsahin@ gmail.com, ORCID: https://orcid.org/0000-0002-6008-4272
} 


\section{Giriş}

Günümüzde kullanılan ve geliştirilmeye devam edilen alfa, beta ve özellikle gama dedektör sistemleri birçok uygulamada önemli kullanım alanlarına sahiptir. Nükleer fizik, parçacık fiziği, nükleer tıp ve diğer uygulamalı alanlar gibi birçok çalışmada ilgilenilen konu hakkında detaylı bilgi alınabilmesi ve doğru değerlendirilmesi için kullanılan dedektörlerin enerji ve zaman çözünürlükleri yanı sıra verimleri de önemlidir. Yapılan deneysel ölçümlerde gerekli olan hassasiyet hem dedektör sistemine hem de kullanılan elektronik sisteme bağlıdır. Nükleer yapı fiziği çalışmalarında dedektör sisteminden istenen önemli özelliklerden birincisi nükleer reaksiyon sonucu ortaya çıkan, hem düşük ve yüksek enerjili gama-ışını ölçümü hem de birbirine yakın enerji değerlerine sahip gama 1şınlarının ayırımının iyi yapılabilmesidir.

Dedektör sistemlerinde istenen ikinci bir özellik ise hassas ve güvenilir ölçümün etki ettiği verim olarak bilinmektedir. Bunların yanı sıra dedektör performansını etkileyen soğutma, dedektör boyutu vb. gibi diğer etkenlerde önemli rollere sahiptir [1]. Özellikle nükleer fizik alanında hem yüksek verimliliğe sahip olan hem de yüksek çözünürlük sağlayan dedektör sistemleri (AGATA, MINIBALL, GAMMASPHERE, GRETA, EXOGAM2, NEDA ve diğerleri gibi) için birçok ülkeden araştırmacılar bir araya gelerek yeni dedektör sistemleri üzerine çalışmalar yapmaktadır $[2,3,4,5,6,7]$. Bu çalışmada deneysel nükleer fizik çalışmalarında uzun süredir kullanılan ve önemli bir konuma sahip olan EXOGAM2 dedektörünün enerji çözünürlüğünün yeni geliştirilen dijital elektronik NUMEXO2 ile nasıl değiştiği bu dijital elektronik ünitedeki K-parametreleri değiştirilerek araştırılmış ve elde edilen sonuçlar bu çalışmada verilmiştir. NUMEXO2 dijital elektronik ünite ile ilgili detaylı bilimsel çalışmalara $[8,9,10]$ referansları aracılığı ile ulaşmak mümkündür.

\section{Materyal ve Metot}

GANIL araştırma merkezinde bulunan hızlandırıcı ile gerçekleştirilen nükleer reaksiyonlar yardımıyla atomik çekirdeklerin uyarılmış durumlarına ve istenildiğinde egzotik çekirdeklere ve bunların uyarılmış durumlarını algılamak için yüksek çözünürlüğe sahip germanyum kristallerinden oluşturulmuş Şekil 1'de gösterilen EXOGAM2 dedektör sistemi kullanılmaktadır. Bu dedektör sisteminde her bir kristal $60 \mathrm{~mm}$ çap ve $90 \mathrm{~mm}$ uzunluğa sahiptir. Bu çalışmada germanyum kristallerinin bir araya getirilmesi ile meydana getirilen EXOGAM2 dedektör sistemi ile yeni NUMEXO2 dijital elektronik sistem birlikte kullanılarak enerji çözünürlüğü ölçümleri gerçekleştirilmiştir. NUMEXO2 dijital elektronik ile veri transferi $100 \sim 200 \mathrm{MHz}$ arasında olmaktadır, analog elektronik sisteminde ise veri transferi yaklaşık olarak 10 20 kHz civarındadır. Özellikle son yıllarda ilgi çeken egzotik çekirdekler için kullanılması planlanan nükleer reaksiyonlarda radyoaktif iyon demeti ve/veya radyoaktif hedef kullanımı gerçekleştirilen nükleer reaksiyon sonucunda aynı anda bir çok reaksiyon kanalı oluşturduğu ve çok kısa zaman dilimlerinde birçok olayı algılayabilme yeteneğine sahip dedektör sistemine ve uygun veri alma hızına sahip elektronik sisteme ihtiyaç duyulmaktadır. İşte bu ihtiyacı giderecek veri alma sistemi EXOGAM2 dedektörü ile birlikte kullanılacak olan NUMEXO2 dijital elektronik ile mümkün olabilmektedir. 


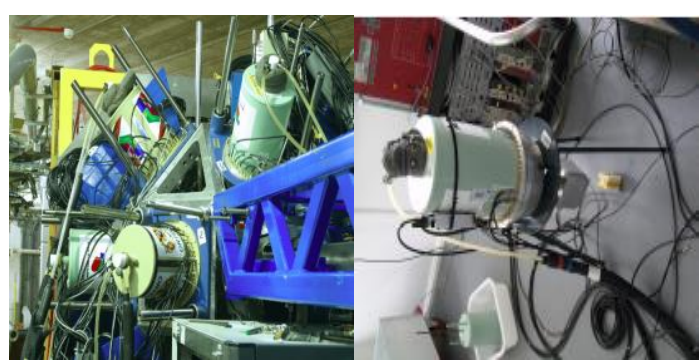

Şekil 1. EXOGAM2 dedektör topluluğunun bir kısmını gösteren fotoğraf (sol) [11]. Testi yapılan bir EXOGAM2 dedektör fotoğrafı (sağ)

$\mathrm{Bu}$ çalışmamızda NUMEXO2 dijital elektronik ünitenin farklı $\mathrm{K}$ parametreleri kullanıldığında dedektör çözünürlüğünde ne tür etkiler yaptı̆̆ını araştırdık. Bunun için bu çalışmada yapılacak olan deney öncesinde dedektör kalibrasyonu için düşük ve yüksek gama enerjilerine $(121,728,344,785,778,904,964,079,1085,869,1112,074,1408,006$ $\mathrm{keV}$ ) sahip ${ }^{152}$ Eu radyoaktif kaynağı kullanıldı. Bilinen bu değerler ile kullanılan dedektör ve elektronik sistemden elde edilen veriler kullanılarak kalibrasyon işlemi gerçekleştirildi. EXOGAM2 dedektöründe yer alan her bir kristal ve daha sonra kristallerin her bir bileşeni ile oluşturulan bir bütün halinde EXOGAM2 dedektörü için enerji kalibrasyon işlemleri ${ }^{152} \mathrm{Eu}$ radyoaktif kaynak kullanılarak elde edilen sinyalin puls yüksekliği spektrumları analiz edilerek incelenmiştir.

Enerji çözünürlüğünü ifade etmek için her bir kristalden elde edilen spektrumda fotopiklerin yarı yüksekliklerinin tam genişliği FWHM (Full-Width Half Maximum) ölçümünü kullanıldı [12]. Şekil 2'de EXOGAM2 dedektöründe germanyum kristalleri kullanılarak, bilinen ve ${ }^{152} \mathrm{Eu}$ kaynağından ortaya çıkan gama sşınları değerleri kullanılarak yapılan kalibrasyon sonucunda ${ }^{152}$ Eu radyoaktif kaynağından elde edilen enerji spekturmu gösterilmektedir. Şekil 2'de gösterilen farklı renkler dedektör sistemi (birinci EXOGAM2 ve ikinci EXOGAM2) içinde yer alan ve her bir kristal için farklı bileşenlerinden elde edilen piklerin kalibrasyon işlemi sonucu ilgili gama 1şınında gösterilmesini ifade etmektedir.
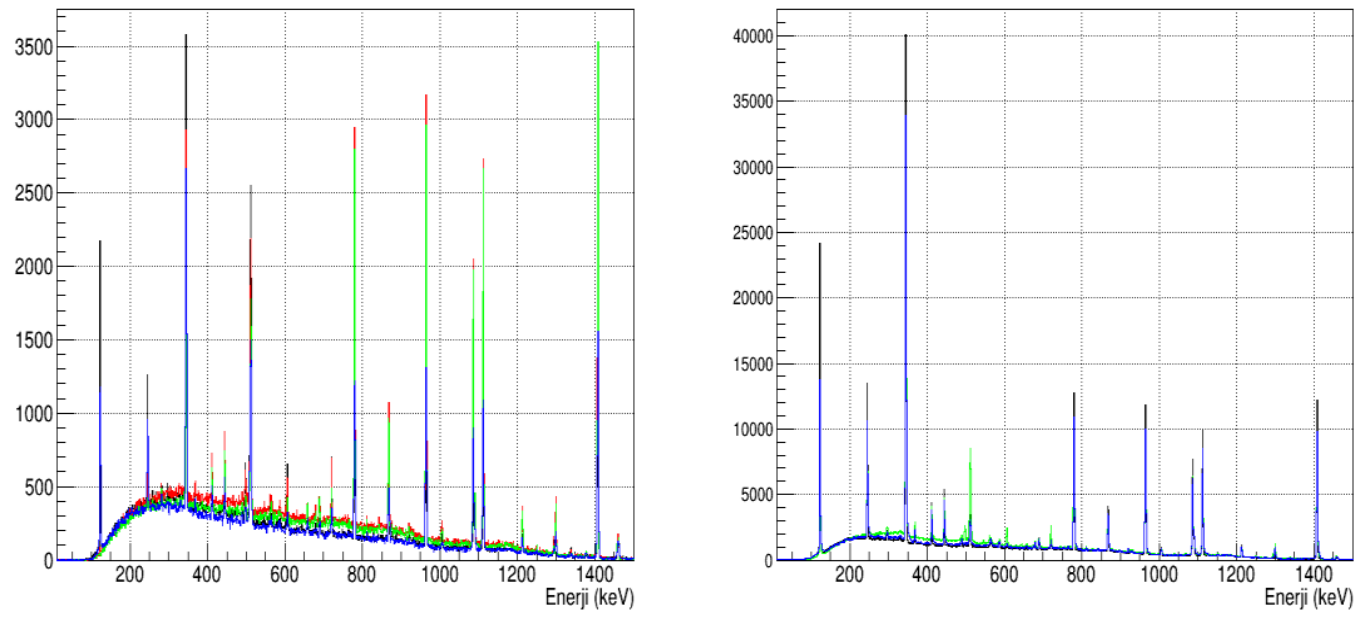

Şekil 2. EXOGAM2 dedektörü için ${ }^{152}$ Eu kaynağından elde edilen enerji dağılımı birinci EXOGAM2 (sol) ikinci EXOGAM2 (sağ) 


\section{Bulgular}

Kalibrasyon için kullanılan ${ }^{152}$ Eu radyoaktif kaynak ile kaydedilen verilerin analizinden elde edilen kalibrasyon katsayıları her bir EXOGAM2 dedektörü için kullanılarak ilgilenilen düşük ve yüksek enerjili gama enerji pikleri ilgili konumlarına getirilmiştir. EXOGAM2 dedektör sisteminin enerji çözünürlüğünü incelemek için, düşük ve yüksek enerjili gama 1şınlarına sahip olan ${ }^{152}$ Eu kaynağından elde edilen spektrumların ilgili pikleri içeren kısımları, Şekil-3'de gösterilmektedir. Her bir spektrum için Gauss fonksiyonu kullanılarak fit işleminin uygulanması ile dedektör enerji çözünürlük değerleri elde edilmiştir.
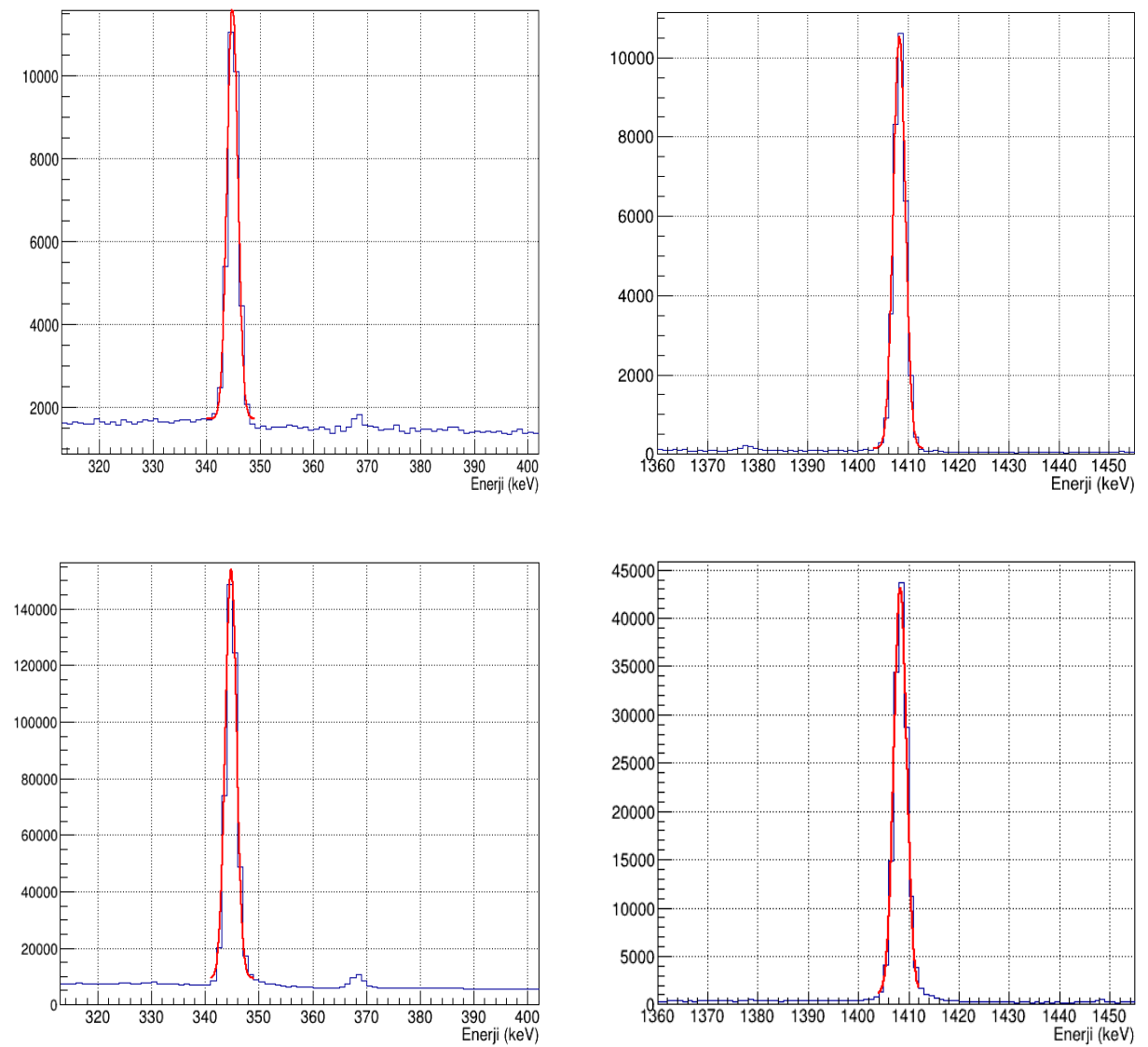

Şekil 3. Birinci EXOGAM2 (üst), ikinci EXOGAM2 (alt) dedektörleri için düşük $(344,278$ keV) (sol) ve yüksek enerjili (1408,006 keV) (sağ) enerji pikleri

Tablo 1'de ${ }^{152} \mathrm{Eu}$ kaynağından elde edilen gama 1şınlarının, EXOGAM2 dedektöründen elde edilen ve her bir kristalin bileşeni için enerji çözünürlük değerleri ve bu kristallerin birlikte oluşturduğu dedektör toplamı için enerji çözünürlük değerleri gösterilmektedir.

Tablo 1. ${ }^{152}$ Eu kaynağından elde edilen enerji çözünürlük değerleri

\begin{tabular}{lcc}
\hline Kristal numarası & $\begin{array}{c}\mathbf{3 4 4 , 2 7 8 ~ k e V} \\
\text { FWHM }\end{array}$ & $\begin{array}{c}\mathbf{1 4 0 8 , 0 0 6 ~ k e V} \\
\text { FWHM }\end{array}$ \\
\hline 1. EXOGAM2-1.Bileşen & 2,094 & 2,669 \\
1. EXOGAM2-2.Bileşen & 2,184 & 2,723 \\
1. EXOGAM2-3.Bileşen & 2,049 & 2,665 \\
1. EXOGAM2-4.Bileşen & 3,212 & 3,605 \\
1.EXOGAM2(toplam) & 2,357 & 2,820 \\
\hline 2. EXOGAM2-1.Bileşen & 2,366 & 2,841 \\
2. EXOGAM2-2.Bileşen & 2,232 & 2,763 \\
2. EXOGAM2-3.Bileşen & 2,801 & 3,257 \\
2. EXOGAM2-4.Bileşen & 2,615 & 3,153 \\
2.EXOGAM2(toplam) & 2,451 & 2,975 \\
\hline
\end{tabular}


$\mathrm{K}=2 \mu \mathrm{s}, \quad \mathrm{K}=5 \mu \mathrm{s}$ ve $\mathrm{K}=10 \mu \mathrm{s}$ parametreleri için elde edilen dedektör enerji çözünürlük değerleri ise Tablo 2'de gösterilmektedir.

Tablo 2. Farklı K-parametreleri kullanılarak ${ }^{152}$ Eu kaynağından elde edilen enerji çözünürlük değerleri

\begin{tabular}{|c|c|c|}
\hline $\begin{array}{c}\mathrm{K}=2 \mu \mathrm{s} \\
\text { Kristal numarası }\end{array}$ & $\begin{array}{c}344,278 \mathrm{keV} \\
\text { FWHM }\end{array}$ & $\begin{array}{c}\text { 1408,006 keV } \\
\text { FWHM }\end{array}$ \\
\hline 1. EXOGAM2-1.Bileşen & 2,431 & 2,532 \\
\hline 1. EXOGAM2-2.Bileşen & 2,597 & 2,770 \\
\hline 1. EXOGAM2-3.Bileşen & 2,668 & 2,662 \\
\hline 1. EXOGAM2-4.Bileşen & 3,176 & 3,111 \\
\hline 1.EXOGAM2(toplam) & 2,743 & 4,171 \\
\hline 2. EXOGAM2-1.Bileşen & 2,704 & 2,719 \\
\hline 2. EXOGAM2-2.Bileşen & 2,743 & 2,380 \\
\hline 2. EXOGAM2-3.Bileşen & 3,525 & 3,046 \\
\hline 2. EXOGAM2-4.Bileşen & 3,671 & 3,438 \\
\hline 2.EXOGAM2(toplam) & 2,756 & 2,530 \\
\hline$K=5 \mu s$ & $344,278 \mathrm{keV}$ & 1408,006 keV \\
\hline Kristal numarası & FWHM & FWHM \\
\hline 1. EXOGAM2-1.Bileşen & 3,076 & 3,196 \\
\hline 1. EXOGAM2-2.Bileşen & 3,083 & 3,188 \\
\hline 1. EXOGAM2-3.Bileşen & 2,834 & 3,172 \\
\hline 1. EXOGAM2-4.Bileşen & 3,793 & 4,042 \\
\hline 1.EXOGAM2(toplam) & 3,069 & 4,091 \\
\hline 2. EXOGAM2-1.Bileşen & 3,588 & 3,156 \\
\hline 2. EXOGAM2-2.Bileşen & 3,041 & 3,492 \\
\hline 2. EXOGAM2-3.Bileşen & 3,579 & 2,930 \\
\hline 2. EXOGAM2-4.Bileşen & 4,187 & 3,581 \\
\hline 2.EXOGAM2(toplam) & 4,187 & 3,581 \\
\hline$K=10 \mu \mathrm{s}$ & $344,278 \mathrm{keV}$ & $1408,006 \mathrm{keV}$ \\
\hline Kristal numarası & FWHM & FWHM \\
\hline 1. EXOGAM2-1.Bileşen & 2,573 & 2,841 \\
\hline 1. EXOGAM2-2.Bileşen & 2,397 & 2,937 \\
\hline 1. EXOGAM2-3.Bileşen & 2,535 & 2,806 \\
\hline 1. EXOGAM2-4.Bileşen & 3,882 & 4,389 \\
\hline 1.EXOGAM2(toplam) & 3,029 & 3,238 \\
\hline 2. EXOGAM2-1.Bileşen & 2,632 & 2,925 \\
\hline 2. EXOGAM2-2.Bileşen & 3,564 & 2,693 \\
\hline 2. EXOGAM2-3.Bileşen & 3,891 & 2,897 \\
\hline 2. EXOGAM2-4.Bileşen & 3,703 & 2,902 \\
\hline 2.EXOGAM2(toplam) & 3,715 & 2,874 \\
\hline
\end{tabular}

\section{Sonuç ve Yorum}

Yapılan bu çalışmanın amacı EXOGAM2 dedektörü ile birlikte yeni geliştirilen NUMEXO2 dijital elektronik sisteminin, ${ }^{152} \mathrm{Eu}$ radyoaktif kaynağının kullanılması ile enerji çözünürlüğü yönünden performansının incelenmesi olmuştur. Yapılan bu enerji çözünürlüğü ölçümü ile kullanılan dedektör sistemi ve elektronik sistemin yapılacak olan bir deneysel çalışma ile ne gibi neticeler elde edileceği deney yapılmadan önce göz önüne getirilmekte ve gerekli olan durumlarda dedektör ve kullanılan elektronik sistem üzerinde iyileştirmeler yapmak için önem arz etmektedir. Yapılan bu enerji çözünürlügü ölçümünde ${ }^{152}$ Eu kaynağından elde edilen düşük ve yüksek enerjili gama ışınlarından elde edilen enerji seviyeleri dikkate alınmıştır. Gerçekleştirilen deneysel çalışma ile elde edilen verilerin analiz sonucu herhangi bir K parametresi uygulanmadan birinci EXOGAM2 dedektörü için FWHM ile ifade edilen çözünürlük değerleri sırası ile $(344,278 \mathrm{keV})$ için 2,357 ve $(1408,006 \mathrm{keV})$ için 2,820 olarak ölçülürken ikinci EXOGAM2 dedektörü için ise FWHM 
değerleri sırasıyla 2,451 ve 2,975 olarak ölçülmüştür. Farklı K-parametrelerinin uygulanması ile $\mathrm{K}=2 \mu \mathrm{s}$ için bu değerler birinci EXOGAM2 dedektöründe düşük ve yüksek gama enerjileri için FWHM değerleri sırasıyla 2,743 ve 4,171, ikinci EXOGAM2 dedektörü için ise sırası ile 2,756 ve 2,530 olarak ölçülmüştür. Aynı işlemler $\mathrm{K}=5 \mu$ s için yapıldığında birinci EXOGAM2 dedektöründe düşük ve yüksek gama enerjileri için sırasıyla FWHM değerleri 3,069, 4,091 ve ikinci EXOGAM2 dedektörü için sirasıyla 4,187 ve 3,581 olarak ölçülmüştür. Son olarak $K=10 \mu \mathrm{s}$ için birinci EXOGAM2 dedektöründe düşük ve yüksek gama enerjileri için FWHM değerleri sırasıyla 3,029, 3,238 ve ikinci EXOGAM2 dedektörü için ise sırasıyla 3,715 ve 2,874 olarak elde edilmiştir.

Elde edilen enerji çözünürlük değerleri incelendiğinde özellikle $K=2 \mu$ s parametresi için düşük gama enerjilerinde elde edilen spektrumun iyi bir çözünürlük değerine sahip olduğu görülmektedir.

\section{Araştırmacıların Katkı Oranı Beyanı}

Vakkas Bozkurt: Araştırma, Analiz, Orjinal Taslak Yazımı, Veri İyileştirme.

Sefa Ertürk: Analiz, Veri iyileştirme, İnceleme ve Düzenleme, Malzeme Temini, Tavsiye.

Elif Şahin: Gözlem, İnceleme ve Düzenleme.

\section{Destek ve Teşekkür Beyanı}

Bu çalışma TÜBITTAK 114F473 nolu proje trafında desteklenmiştir bu destek için TÜBİTAK'a teşekkür ederiz. Ayrıca bu çalışmayı gerçekleştirdiğimiz GANIL çalışanları Gilles de FRANCE, Michel TRIPON, Frederric SAILLANT ve diğer laboratuvar çalışanlarına teşekkür ederiz.

\section{Çatışma Beyanı}

Bu çalışmanın yazarları olarak herhangi bir çatışma beyanımız bulunmadığını bildiririz.

\section{Etik Kurul Onayı ve/veya Aydınlatılmış Onam Bilgileri}

$\mathrm{Bu}$ çalışmanın yazarı olarak herhangi bir etik kurul onayı ve/veya aydınlatılmış onam bilgileri beyanımın bulunmadığını bildiririm.

\section{Kaynakça}

[1] R. H. Pehl, "Radiation damage of germanium detectors," in Proc. Gamma Ray Spectroscopy in Astrophysics Symposium, Greenbelt, Maryland, 1978, pp. 1-10.

[2] G. de Angelis, A. Bracco, and D. Curien, "The EUROBALL gamma ray detector spectrometer," Europhysics News, 34 (5), 181-185, 2003.

[3] F. S. Goulding, D. A. Landis, N. Madden, M. Maier, and H. Yaver Goulding, "GAMMASPHEREOverview of detector and signal processing system," in Proc. IEEE Nuclear Science Symposium and Medical Imaging Conference Record, San Francisco, 1995, pp. 432-436.

[4] J. Erberth et al., "Miniball: A gamma-ray spectrometer with position-sensitive Ge detectors for nuclear structure studies at REX-ISOLDE", in Proc. Conference of Frontiers of Nuclear Structure, Berkeley, California, 2003, pp. 349-356.

[5] G. de France, "High resolution gamma-ray spectroscopy at GANIL,in Proc. 36th Brazilian Workshop on Nuclear Physics, Sao Paulo, 2014, pp. 10-16.

[6] S. Akkoyun et al., "AGATA - Advanced gamma tracking array," Nucl. Instrum. Methods Phys. Res. A, 668, 26-58, 2012.

[7] C. W. Beausang et al, "GRETA. The gamma-ray energy-tracking array. Status of the development and physics opportunities," Nucl. Instr. and Meth. B, , 204, 666-670, 2003.

[8] F. J. Egea et al.. "A new front-end high-resolution sampling board for the new-generation electronics of EXOGAM2 and NEDA detectors," IEEE Transactions On Nuclear Science, 62 (3), 1056-162, 2015.

[9] F. J. Egea, et al., "Digital front-end electronics for the neutron detector NEDA," IEEE Trans. Nucl. Sci., $62(3), 1063-1069,2015$. 
[10] X. Egea et al., "Design and Test of a High-speed Flash ADC Mezzanine Card for High-resolution and Timing Performance in Nuclear Structure Experiments", in Proc. 18th Real-Time Conference, Berkley, 2012.

[11] G. de France. (2020, May 27). EXOGAM detectors. Available: https://www.ganilspiral2.eu/scientists/ganil-spiral-2-facilities/instrumentation/exogam

[12] G. F. Knoll, Radiation Detection and Measurement. New York: John Wiley \& Sons, Inc., 2010, 830p. 